\title{
Study on the dynamic response characteristics of a fuse under the excitation of the detonation field
}

\author{
Weiguang Zhang ${ }^{1, a}$, Xingyong Gao ${ }^{1}$,Yuling Zhang ${ }^{1}$, Xiaofeng $\mathrm{Li}^{2}$,Shuaijie $\mathrm{Liu}^{2}$ \\ ${ }^{1}$ Ordnance Engineering College, Shijiazhuang 050003,China \\ ${ }^{2}$ Beijing Institute of Technology, Beijing 100081,China \\ a15614107072@163.com
}

Keyword: tandem warhead, detonation field, fuse, arming environmental.

\begin{abstract}
In this paper, the research status of the tandem warhead at home and abroad is reviewed.The arming types and the corresponding principles for the fuse are introduced.The present situation and the research emphasis of the research on the influence of the front detonation field on the rear - level are analyzed. The analysis shows that in the design of the series warhead, it is a hotspot in the field of arming environmental to make full use of the environmental force of the front stage detonation field as the fuse. It is also a research direction in the design of following fuse in the future.
\end{abstract}

\section{Introduction}

In the modern war, the ground high value targets (such as the command post, fire point), underground command center, ammunition depots, the aircraft base is generally located in the reinforced concrete structure. Reinforced concrete as a kind of important construction materials, with the good performance of fast and convenient making, high compressive strength, fire resistance, anti corrosion, tensile resistance, which is widely used in civil and military fields. To deal with this kind of solid target, exploded fragments of warhead could not penetrate the layer of reinforced concrete, and the shock wave overpressure damage to reinforced concrete structure is limited. Therefore, to deal with reinforced concrete targets, the most effective way is to make the warhead explode into the internal of the target. The traditional method of damage is to use the penetration blasting warhead, depending on the projectile impact kinetic energy to penetrate into the target and detonate high charge in the warhead to destroy the target. In order to achieve the ideal damage effect, the quality of warhead is correspondingly larger and need larger aircraft to mount, at the same time, it demands the higher attack speed. In addition, when the impact angle and the angle of attack is large, it is prone to ricochet which will not penetrate the target effectively. It is urgent to develop a new type of warhead, which can effectively kill the target of concrete structures, that is, tandem assault warhead. Compared with the kinetic energy penetrator warhead, the weight of tandem warhead is lighter, and increase the impact angle range, which is more suitable for added to standard weapons, so a lot of active service and equipment in development are equipped with the tandem warhead.

Tandem warhead generally contains two-stage constitution. The forward charge explode firstly to form the metal jet or high-speed shaped charge penetration to open holes on the concrete target and its overlying soil layer, the rear intrude into the internal of the warhead and explode to achieve the effective destruction of the target ${ }^{[1]}$.In order to ensure the damage effect of warhead, it is necessary to guarantee the reliability of fuse. fuse failure often leads to the failure of the warhead or the security problem, which affects the fighting force. So it is very important to study the dynamic response of the fuse under the condition of the detonation field. Especially the dynamic response of the rear - level fuse in the environment of the first - stage detonation has become a hot research topic at home and abroad. 


\section{The domestic and international development situation of tandem warhead}

\subsection{The international development situation of tandem warhead.}

In the early 1980s, LLNL laboratory in the US first carried out the research of the two levels tandem penetration warhead ${ }^{[2]}$. In the 1990 s series penetration warhead began to be applied to a variety of weapon platform. The first multistage penetration warhead in army is SG-357 anti runway munitions. It is a part of the JP233 payload dispenser carried by RAF Tornado fighter. In the Iraq war, the U.S. military has used the AGM-114 Hellfire missile with tandem penetration warhead to attack small bunker ${ }^{[3]}$. Broach jointly developed by Royal Ordnance Department of British aerospace company, Thomson-thorn missile electronic equipment and Defense Evaluation\& Research Bureau, which uses two levels of penetration structure. The front part shaped charge penetrating hard target surface, and the $245 \mathrm{~kg}$ high explosive bombs in the rear part penetrate the enhanced bunker, exploded inside the target, it can destroy reinforced concrete targets of $6 \mathrm{~m}$ thick. The penetration ability is 2 times of the ordinary bomb. They prepare to install it to American AGM-154C JSOW, the second or the third generation paveway laser guided bombs and American Tomahawk cruise missiles. Lancer, jointly developed by Britain, the US and Italy, comes from small spear warhead. It carried by the Jaguar fighter, for drill ground bomb at least $450 \mathrm{~kg}$ heavy, can also be installed to air missiles on Hawke. Mephisto, TDA/TDW Company began to develop since 1997, is used to the KEPD-150/KEPD-350 penetration warhead. The front part of the intelligent Mephisto is equipped with photoelectric detector proximity fuse and the penetration warhead in the rear part contains a program of multipurpose fuse system in the tail, it maintains 3 kinds of operating modes include setting airburst, trigger initiation and trigger delay initiation. Under the penetration mode, the sensor can be used to detect the layer of the target. The warhead drill through multilayer structure such as sand and concrete, then explode in the bunker inside according to the trigger signal which procedures set on the preset point.

In addition, the French KRISS penetration projectile also adopts penetration warhead structure. Foreign countries have developed tandem penetration ammunition for individual weapon system such as the German $110 \mathrm{~mm}$ rocket projectile. The rocket use the German Panzerfaust III of $60 \mathrm{~mm}$ anti tank rocket to launch and the rocket warhead consists of shaped charge, concomitant bullets and corresponding fuse. When the warhead hit the target, shaped charge produces a hole with $50-60 \mathrm{~mm}$ diameter in the wall of a target, then the concomitant bullets with electric shock delay fuse through the hole at nearly same speed with warhead and explodes about $1-2.5 \mathrm{~m}$ behind the goal. The concomitant bullet has more than 1400 spherical fragment. The infantry potent hollowprojectile, began to develop in 1988, equipped in American special forces in 1997. Its main task is to destroy the reinforced concrete bridges and other types of strong works. This ammunition is composed of two parts. The front part adopts EFP warhead, the purpose is to get a larger hole, which can help second warhead to explode. It contains $200 \mathrm{~mm}$ diameter, $13.6 \mathrm{~kg}$ weight, $675 \mathrm{~mm}$ length. The bomb can destroy the reinforced concrete pier with $1.8 \mathrm{~m}$ diameter.

\subsection{The domestic development situation of tandem warhead.}

The development of tandem warhead in our country begin quit late, Though the efforts of scientists and technicians, they has basically solved the key technical problems in the design of the tandem warhead, and completed the primary design of a certain type of tandem charge warhead. The tandem warhead can not only penetrate the NATO three spaced heavy targets with explosive reactive armor under the mode of static penetration and simulated penetration, but also can penetrate it without reaction armor ${ }^{[4]}$. China's Red Arrow 9 anti tank missile warhead adopts two parts of tandem structure, mainly composed by pre charge and main charge ammunition. The main charge ammunition adopts double tapered copper liner。The telescopic double fried high stick in the front of tandem warhead usually bound and nested together by the launch tube. Under the spring force the fried high rod get out and locked after launch. In addition, install non metallic material between the front and back charge ammunition to further protect the rear - mounted charge to avoid the impact of the explosive charge of the first - scale ${ }^{[5]}$.

A new generation of Chinese infantry anti tank weapons- PF98 $120 \mathrm{~mm}$ anti tank rocket have been used formal armed forces in 1999. At 1st August in 2000, its debut was at the Hong Kong 
Garrison weapons show.In order to deal with the suit of armor explosive reactive armor battle tanks, the anti tank rocket warhead is also used in the tandem warhead technology, the tandem warhead technology can effectively breakdown armor explosive reactive armor NATO heavy three layer target.

\section{Fuse research on the solution preserving property}

Fuse safety mechanism is the most basic one, which plays an important role in the whole security system $^{[6]}$. There are three kinds of fuse safety mechanism, which is the mechanical insurance mechanism, the mechanical and electrical safety mechanism and the electronic insurance mechanism $^{[7]}$.

\subsection{Mechanical insurance mechanism.}

In the early stage of mechanical fuse, the safety element in fuse is used as the sensitive element, which is used in the inertia force of the launching process. Under the action of inertia force, the safety element can overcome the effect of the restriction element, release the separation explosive or release the restriction to the firing mechanism in order to free fuse. Setback arming device is the typical structure, they are mainly elastic setback arming device and rigid setback arming device. Both of them have the advantages of simple structure and reliable performance ${ }^{[8]}$. In addition, there are insurance institutions, zigzag slot card mechanism, interlocking two degrees of freedom setback mechanism etc. In the mechanical insurance institution, most of the foreign countries use the watch mechanism to realize the release solution. In the early stage of the Soviet Union, they used delay powder to realize the lock. On mechanical insurance device, a lot of research works have been carried out in the domestic. Such as Li Laifu etc in Nanjing University of science and technology ${ }^{[9]}$, they analyze the response characteristic of the fuse setback arming device and rigid elastic recoil safety structure when facing different recoil overload time. And find the release time response of two kind of setback insurance mechanism under the different recoil overload time. Cao Ying ${ }^{[10]}$ etc in Nanjing University of science and technology carried out the research on the theory of fuse setback arming device with two degrees of freedom. They analyzed the motion process of the fuse setback arming device with two degree of freedom and theoretically proves the feasibility of the application of the fuse setback arming device with two degree of freedom in $100 \mathrm{~g}$ of low launch overload environment. Wang Xiaoxia ${ }^{[11]}$ etc in Xi'an electrical information institute proposed a working MEMS setback mechanism under low emission overload situation. It can ensure the rocket fuse safety in service management encountered in the process of falling accident, and the release insurance of rocket fuse reliability in the force of low overload launch environment. As the mechanical insurance mechanism have energy itself under inertia force, it can be directly used in the safety parts and working in the predetermined rules. Simple design as well as mature technology of the mechanical insurance mechanism, it has been wide used in the modern fuse especially in the progress of launching overload ammunition fuse.

\subsection{Mechanical and electrical safety mechanism.}

The mechanical and electrical safety mechanism uses the environmental sensor to replace the mechanical environment sensitive device. To realize the fuse environment detection, and the driver of the release insurance equipment generally adopt electric initiating device at the same time. The mechanical and electrical safety mechanism has better recognition ability to the environment, and it is more perfect in logic and function of the insurance control. After recognizing of the environmental information, and then transmit to the executive body, it can release insurance. Mechanical and electrical mechanism contains safety and reliability, and it is the mainstream of the fuse development. The foreign research of the mechanical and electrical insurance institution began in 1970, and in 1980 it had been equipped in the standard fuse, such as M934E5 fuse. The most used in the US like MK432MOD0 and XM762A1 fuse are also used the mechanical and electrical mechanism. Mechanical and electrical insurance mechanism is also the current domestic research direction such as Han Jie ${ }^{[12]}$ etc in the Beijing Institute of Technology. They design a kind of mechanical and electrical safety mechanism for fuse under low environmental force. This mechanism uses the acceleration sensor as the detection element, and single chip microcomputer as 
the control element. The acceleration sensor is used to detect the axial and the centrifugal acceleration which are the condition of releasing insurance. Ma Shaojie ${ }^{[13]}$ etc in Nanjing University of Science and Technology proposed lubrication design of small caliber fuse of electromechanical safety mechanism. They solved the contradiction between the output torque and the driving torque required force when micro-machine used in the small caliber projectile fuse.

\subsection{Electronic insurance mechanism.}

Electronic insurance mechanism is a new type of fuse safety system based on the serial explosion sequence. It adopts the explosive sequence of initiating explosive devices which used the insensitive explosive, by controlling the ignition energy supply to ensure the safety of fuse. The control of security system does not require mechanical isolation, and it is widely used in high value Ammunition Fuse. But on the whole, there is still less applications in the research stage of conventional ammunition fuse.

\section{Study on the incentive effect of the front stage detonation field to the rear}

\subsection{Foreign research status.}

On the study of the incentive effect of the front stage detonation field to the rear, the foreign mainly focus on the matching relationship of the front with the rear. Early 1980s, Murphy ${ }^{[9]}$ and other scholars began to carry out the correlation study of the front charge penetration with the rear stage kinetic energy projectile penetrating into concrete, and the influence of the front stage detonation field on the rear - stage kinetic energy projectile is analyzed, and the efficiency relationship of the front charge penetration with the rear stage kinetic energy projectile was established, which was the foundation of the research on the relationship between the front and the rear. Malygin ${ }^{[14]}$ and so on carried out the deep research on the initiation mode of the front and rear stage of the tandem warhead, and studied a warhead which is reverse priming type and tandem shaped charge structure based on the X optical test. Held ${ }^{[15]}$ et al. studied and designed the front charge structure, by means of numerical simulation and experiment, the relationship between the structure of the charging structure, the opening performance, the hole quality and the matching of the front and the rear is studied.

\subsection{Domestic research status.}

Based on the rapid development of the tandem warhead, in order to pursue the better damage effect, but also to improve the design of the warhead, the domestic universities, research institutes, factories and other institutions has pay more and more attention to the relationship of the front and rear stage, and has made a series of research results.

The Zeng Biqiang and Jiang Chunlan ${ }^{[16-17]}$ of Beijing Institute of Technology studied the influence of the pre detonation velocity, attitude and projectile structure on the rear velocity, attitude and projectile structure on the situation when the axis of the projectile is not coincident with the full elastic shaft of the tandem warhead by the method of three-dimensional numerical simulation. The change law of the impact of different initial velocity of the projectile, the lateral deflection of the projectile, the attitude of the projectile, and the mechanical response of the projectile with the explosive charge were studied. Along with the view that with initial velocity with into the bomb before the blast, the ballistic side partial and attitude overturning effect can be greatly reduced. But the impact of the projectile and the structural response is not obvious, which provides a reference for the design of the related tandem warhead.

Wang Jian, Ruan Wenjun ${ }^{[18]}$ of Nanjing University of Science and Technology use Autodyn software, the explosive field of demolition-blast traveling hard structural tandem warhead was investigated by numerical simulation. The blast pressure, velocity and temperature distributions were gained. The response blast shock wave pressures varied against time of sub-munition fore-body and charge were calculated. The explosive overload and velocity drop-off of sub-munition were obtained. Simulation results show that sub-munition charge has sympathetic detonation security and enough traveling velocity, and explosive overload can meet sub-munition fuse arming demand.

Duan Zhenlong, Yuan Pengju ${ }^{[19]}$ etc. aim at the lack of arming environment for following fuse of 
tandem warhead, a method of following fuse arming using precursory detonation was pet forward. The method used the shock wave produced by precursory detonation to make shear pin cut the copper wire. Experiments results showed that the following fuse could make use of the precursory charge as an arming environment, which was testified by theoretical analysis and simulation results.

Chen Huiwu, Zhang Xianfeng ${ }^{[20]}$ of Nanjing University of Science and Technology carried out a three-dimensional computational study of blast effect on traveling projectile of tandem warhead and has been performed using finite element programme of LS-DYNA. Numerical simulation of different distance of two-stage and different initial velocity has been carried out overpressure and velocity drop of traveling projectile has been got based on different initial conditions. Essential analysis and conclusion has been derivated at last of the paper, and the computational result has high value to consult for TWPB design.

To study the influence of the tandem warhead's front charge to following warhead and verify the feasibility of installing annular shaped charge in the front of active missile's warhead, the annular cutter's influence to following warhead was simulated by ANSYS/LS-DYNA by Wang Weili, Song Zhiyong ${ }^{[21]}$, etc. of Naval Aeronautical Engineering Institute. The date of acceleration, velocity and displacement, the max stress of following warhead's shell, charge and their place were obtained in different distance between front charge and following warhead. Simulation results showed when following warhead was relating rest to front charge, the shock wave of annular cutter affected following warhead's acceleration, velocity, displacement and pressure of shell and charge, differently in different distance.

Xue Xinying ${ }^{[2]}$ of Nanjing University of Science and Technology has analyzed the influence of the reverse overload of the front stage warhead on the flight speed and the overload of the rear level warhead. The change rule of the flying velocity of the post - level warhead is analyzed and the overload data were obtained and the simulation results were verified by experiments. In addition, Kang Yanlong, Jiang Jianwei and so on ${ }^{[23-24]}$ of Beijing Institute of Technology has carried out the explosive effect of the front charge on the rear stage bullets, using AUTOCYN-2D modeling simulation, the relationship of following level with the kinetic energy of the bullet and the remaining speed and the quality of the non dimensional is obtained; In order to meet the effective matching of the front and rear, Wang Lixia, et al. of Xi'an Modern Chemistry Research Institute studied the penetration of the target plate with different drug loading structures ${ }^{[25]}$.

\section{Summary}

With the improvement and development of the defense system of the underground works, the design of the tandem warhead will be a great demand for the future. Whether to ensure that the series of crucial bombs in the battlefield to hit the target effectively, and to ensure that all the two stages of the battle to achieve the effective penetration of the effect will be a matter of war success or failure.Through this review, We review the current status of domestic and foreign research on the tandem warhead.The research progress on the dynamic response of the fuse under the excitation of the detonation field at home and abroad is analyzed.It can be seen that, although the research on the problem of the dynamic response of the fuse is not much on the study of the relationship between the front and rear level effects in the tandem warhead, as the first stage detonation field can be used as a arming environmental for the fuse, the new viewpoint is put forward, which will become a new development direction in the future research of series battle.

\section{Reference}

[1].Liu Yongyuan,Jiang Zhengping,Zhangjin.Drill Ground Bomb and Its Development Trend [J]. Cruise Missile, 2006,(3):34-37.

[2].Murphy,M.J..Performance analysis of two-stage munitions, 8th International Symposium on Ballistics [C].TB23-29,1984

[3].Wu Xinmin, Wang Zhonghua.Foreign Airborne Weapon Battle Manual[M]. Ordnance Industry 
Press, 2005.

[4].Liu Huansong. The New Development of the Infantry Portable Weapon System [J]. Defence -related Science and Technology,2005(9):12-13.

[5].Xuhui.Air Defense Ship and Anti Aircraft Carrier Battle [J]. World Military, 2006, 12 (1):78 $-82$.

[6].Li Laifu.A Grenade Mechanical Trigger Initiation Research on Key Technology offuse[D].Nanjing:Nanjing University of Science and Technology, 2014.

[7].Zhang Ensu.Simulation and Analysis of fuse Insurance Mechanism Based on ADAMS[D].Taiyuan:North University of China, 2014.

[8].Wang Xiaofu, Shang Yaling. Performance Analysis between fuse Setback Arming Device with Two Degree of Freedom and That with Single Degree of Freedom [J]. Sichuan Ordnance Journal, 2011, 32(6):33-36.

[9].Li Laifu, Wang Yushi. The Response Characteristics of the fuse Setback Arming Device forClassical Overload Time[J]. Sichuan Ordnance Journal,2014,35（7）.

[10].Caoying.Study on the Theory of fuse Setback Arming Device with Double Degree of Freedom[D]. Nanjing University of Science and Technology:Nanjing University of Scienceand Technology,2008.

[11].Wang Xiaoxia,Niu Lanjie,Zhaoxu.MEMS Rocket fuse Setback Arming Device [J]. Science and Technology and Engineering,2011,(11).

[12].Hanjie,Shi Gengchen,Yu Runxiang.Electromechanical fuse Arming Device with Low Environmental Force [J].Journal of Detection and Control,2014,(6).

[13].Ma Shaojie,Du Jiangen,Zhanghe.The Lubricating Design for the Mechatronic Safety and Arming Device of Small Calibre fuse [J].Lubrication and Sealing,2005, (5).

[14]. M alygin , Proskuryakov . Matching of operation of " base-head" composite shaped-chargestages[J].Journal of Applied Mechanics and Technical Physics, 2012,53(1):136-139.

[15].Held M.The shaped charge potential [C] //20th International Symposium on Ballistics.Orlando: [s.n.],2002.

[16].Zeng Biqiang,Jiang Chunlan.Analysis for Effect of Precusory Detonation Field onProjectile Following Course in Tandem Warhead [J].Acta Armamentarii,2010,31(1):162-166.

[17].R.FREY,W.LAWRENCE.Shock Evolution After Shaped Charge Jet Impact and Revelance to Explosive Initiation[J].Int.J.Impact Engng.1995,16(4):563-570.

[18].Wang Jian, Ruan Wenjun. Front-rear Influence Numerical Simulation of Traveling HardStructural Tandem Warhead [J]. Journal of System Simulation,2010,22（9）:2231-2234.

[19].Duan Zhenlong, Yuan Pengju. Method of Following Fuse Arming Using PrecursoryDetonation [J]. Journal of Detection \& Control,2011,33 (1).

[20].Zhang Xianfeng, Chen Huiwu. A Computational Study of Blast Effect onTravelling Projectileof Tandem Warhead [J]. Journal of Projectiles, Rockets, Missiles andGuidence,2006.

[21].Song Zhiyong, Wang Weili. Research by Simylation on Front-Rear DistanceInfluence of Following Warhead [J]. Journal of Naval Aeronautical and Astronautical University,2012,27 (4).

[22].Xue Xinying. Research and realization of the Tandem Warhead for Anti-concrete Munition[D] Nanjing: Nanjing University of Science and Technology,2012.

[23].Kang Yanlong, Jiang Jianwei. Investigation of Explosive Effect to FollowedProjectiles 
byShaped Charge of Tandem Warhead [J]. Chinese Journal of High Pressure Physics,2011,25 (5). [24].Sun Chengwei.Explosive Physics[M].Beijing: Science Press， 2011.

[25].Wang Lixia, Yuan Baohui. Design and Experiment Study on the Precursory Shaped Chargeof Tandem Warhead [J]. Chinese Journal of Explosives \& Propellants,2011,34 (4). 